\title{
Expression of $\beta$-site APP-cleaving enzyme 1 in the hippocampal tissue of an insulin-resistant rat model of Alzheimer's disease
}

\author{
SHIZE LI ${ }^{1}$, NINI WANG ${ }^{2}$, JIYU LOU $^{1}$ and XIAOMAN ZHANG ${ }^{3}$ \\ ${ }^{1}$ Department of Neurology, The Second Affiliated Hospital of Zhengzhou University, Zhengzhou, Henan 450014; \\ ${ }^{2}$ Department of Neurology, Zhengzhou Central Hospital Affiliated to Zhengzhou University, Zhenzhou, Henan 450007; \\ ${ }^{3}$ Department of Neurology, The First People's Hospital of Zhengzhou, Zhengzhou, Henan 450004, P.R. China
}

Received June 16, 2014; Accepted March 16, 2015

DOI: $10.3892 / \mathrm{etm} .2015 .2391$

\begin{abstract}
The aim of this study was to investigate the expression of $\beta$-site APP-cleaving enzyme 1 (BACE1) in the hippocampal tissue of an insulin-resistant rat model, and thereby explore the roles of BACE1 and insulin resistance (IR) in the pathogenesis of Alzheimer's disease (AD). A total of 36 male Sprague-Dawley rats, aged 2 months, were randomly divided into three groups. These were an insulin-resistant (experimental) group, a high fat control group and a blank control group. The cognitive function and behavioral changes of the rats were tested by a Morris water maze experiment. Amyloid $\beta(A \beta)$ deposition was detected by an immunohistochemical method. The expression levels of BACE1 in the rat hippocampal tissues were detected by enzyme-linked immunosorbent assay, western blotting and reverse transcription-quantitative polymerase chain reaction technology. The rats in the experimental group had evident learning and memory impairment, with significantly decreased learning memory. The modeling was successful; in the experimental group, the rats exhibited IR and their glucose metabolism was significantly abnormal. However, there was no characteristic pathology of AD. The expression of BACE1 in the brain tissue of rats in the experimental group was significantly higher than that in high fat control and blank control groups $(\mathrm{P}<0.01)$. In conclusion, the expression of BACE1 in the brain tissue of insulin-resistant rats increased, and IR was indicated to participate in the pathogenesis of AD.
\end{abstract}

\section{Introduction}

Alzheimer's disease (AD), with chronic progressive memory, cognitive and other mental retardation as the main clinical

Correspondence to: Dr Jiyu Lou, Department of Neurology, The Second Affiliated Hospital of Zhengzhou University, 2 Jingba Road, Zhengzhou, Henan 450014, P.R. China

E-mail: jiyulou@yeah.net

Key words: Alzheimer's disease, $\beta$-site APP-cleaving enzyme 1, insulin resistance, expression manifestations, is an age-related neurodegenerative disease of the central nervous system. The typical pathological changes of $\mathrm{AD}$ include the appearance of a large amount of senile plaques due to the accumulation of amyloid $\beta(\mathrm{A} \beta)$ in the brain, neurofibrillary tangles formed by the abnormal phosphorylation of $\tau$ protein, and neuronal loss (1). BACE1 is a cleaving enzyme that generates $A \beta$ protein, which plays a role in the pathogenesis of AD. Sporadic AD (SAD) accounts for $95 \%$ cases of $\mathrm{AD}$, but its pathogenesis is not completely clear $(2,3)$. Recent studies have shown that AD may be associated with insulin metabolism and signal transduction $(4,5)$. Insulin resistance (IR) and hyperinsulinism may play a role in the occurrence and development of SAD (6).

In the present study, a rat model of IR was used to observe the pathological changes and expression of BACE1 in the brain tissues of insulin-resistant rats, and thereby investigate the roles of BACE1 and IR in the pathogenesis of AD. The results may provide a theoretical basis for the clinical prevention and treatment of AD.

\section{Materials and methods}

Animal grouping. A total of 36 male Sprague-Dawley rats, 2 months of age, were provided by the Animal Experimental Center of Zhengzhou University (Zhengzhou, China). All the experimental rats were numbered. Twelve rats were selected as the blank control group using a random digits table. They were fed a normal diet. The remaining 24 rats formed the high fat, high sugar and high protein group. They were fed a high fat, high sugar and high protein diet [percentage of calories: $26 \%$ from carbohydrates, $15.12 \%$ from protein and $58.18 \%$ from fat (refined pig oil)] (7). The IR index was calculated 3 months later by the homeostatic model assessment (HOMA) method: IR = fasting blood glucose $x$ plasmatic insulin/22.5). According to the IR index, the 24 rats were divided into a high fat control group and an experimental group (each $n=12$ ). The rats in the experimental group were resistant to insulin (a mean of IR value of 3 standard deviations greater than that in the blank control group was considered to indicate IR). This study was carried out in strict accordance with the recommendations in the Guide for the Care and Use of Laboratory Animals of the National Institutes of Health (Eighth Edition, 2010). The animal use protocol has been reviewed and approved by the Institutional 
Animal Care and Use Committee (IACUC) of Zhengzhou Central Hospital Affiliated to Zhengzhou University.

Establishment of the animal model. Streptozotocin (STZ; $60 \mathrm{mg} / \mathrm{kg}$ ) in citric acid buffer and an equivalent amount of citric acid buffer were slowly injected into the bilateral lateral ventricles of the rats in the experimental group and the high fat control group, respectively. Three weeks later, the cognitive function and behavioral changes of the rats were tested with a Morris water maze test. The rats were then decapitated and the hippocampi were removed and placed on ice.

Morris water maze test. The Morris water maze test was carried out as previously described (8). The latency and number of times the rats crossed the platform were measured in an XR-XM101 water maze apparatus purchased from Shanghai Xinruan Technology Co., Ltd. (Shanghai, China).

Immunohistochemical analysis. Rats from each group were sacrificed after modeling. The brain tissue was excised, embedded with paraffin and cut into $5-\mu \mathrm{m}$ sections. Following dewaxing and hydration of the sections, endogenous catalase was inactivated by treatment with $3 \%$ hydrogen peroxide. The tissue was then blocked with $10 \%$ goat serum for $1 \mathrm{~h}$. Monoclonal rabbit anti-A $\beta$ primary antibody (1:500; Abcam, Cambridge, UK) was applied to the slides, which were incubated overnight at $4^{\circ} \mathrm{C}$ and washed with phosphate-buffered saline with Tween 20 (PBST) three times the next day, for 5 min each time. The slides were then incubated with goat anti-rabbit IgG polyclonal antibody (1:100; \#554020; BD Biosciences, New Jersey, USA) at room temperature for $1 \mathrm{~h}$, washed with PBST three times, for 5 min each time, and counterstained with hematoxylin. The brain tissue was observed under a MM-60 microscope (Olympus, Tokyo, Japan).

Enzyme-linked immunosorbent assay (ELISA). A $100 \mathrm{mg}$ portion of the rat hippocampus was isolated by excision and, in a ratio of $20 \mathrm{mg} / 200 \mu \mathrm{l}$, the corresponding volume of cell lysis buffer $(50 \mathrm{mM}$ Tris $\mathrm{pH} 7.4,250 \mathrm{mM} \mathrm{NaCl}, 0.5 \%$ Triton X100, 10\% glycerol and $1 \mathrm{mM}$ DTT; Roche, Indianapolis, IN, USA) was added to the brain mass. The tissue was homogenized in an ice bath with ultrasonic irradiation. After $30 \mathrm{~min}$, the homogenate was centrifuged for $15 \mathrm{~min}$ at $15,000 \mathrm{x}$ g and $4^{\circ} \mathrm{C}$. The supernatant was extracted in order to determine the concentration of protein. The protein was assayed using a BACE1 ELISA kit (Roche, Indianapolis, IN, USA) according to the manufacturer's instructions. All experiments for each sample were performed in triplicate.

Western blot analysis. The sample was added to the supernatant extracted as described above, $5 \mathrm{X}$ loading buffer was added and the mixture was boiled for $10 \mathrm{~min}$, then centrifuged at $15,000 \mathrm{x}$ g for $5 \mathrm{~min}$. The proteins were extracted, subjected to SDS-PAGE and then transferred to a nitrocellulose membrane, where they were blocked with $5 \%$ bovine serum albumin in phosphate-buffered saline Tween-20 solution for 30 min. Rabbit anti-human BACE1 polyclonal antibodies (\#L4391; Wuhan Boster Biological Technology, Ltd., Wuhan, China) diluted with Tris-buffered saline (TBS) and Tween 20 (TBST; 1:2,000) were added, and the membrane was incubated overnight at $4{ }^{\circ} \mathrm{C}$. After rinsing, goat anti-rabbit IgG polyclonal antibodies (\#554020; BD Biosciences, Franklin Lakes, NJ, USA) diluted with TBST (1:100) were added and the membrane was incubated at room temperature for $1 \mathrm{~h}$. After rinsing, the membrane was stained with DAB in the dark. When the color was developed satisfactorily, TBS was added to terminate the reaction. The strips of film were analyzed using a gel image analyzer (Tocan 240; Tocan Biotechnology Co., Shanghai, China). $\beta$-actin (with TBST, dilution 1:500) was used as the internal reference protein.

Reverse transcription-quantitative polymerase chain reaction (RT-qPCR). A 100-mg quantity of frozen hippocampal tissue was excised in order to extract the total RNA. cDNA was synthesized by reverse transcription using a First Strand cDNA Synthesis kit with M-MLV reverse transcriptase (Takara, Dalian, China). The thermal cycling conditions were $65^{\circ} \mathrm{C}$ for $5 \mathrm{~min}, 25^{\circ} \mathrm{C}$ for $5 \mathrm{~min}, 42^{\circ} \mathrm{C}$ for $60 \mathrm{~min}$ and $70^{\circ} \mathrm{C}$ for $5 \mathrm{~min}$. The cDNA synthesis product $(0.5 \mu \mathrm{l})$ was amplified by PCR. For BACE1, the upstream primer F1 was 5'-CCTCCG AAAGTCTGTGTATGTG-3', the downstream primer R1 was 5'-CAGTTGGAACCATTGATGAAGA-3', and the product was a fragment of $159 \mathrm{bp}$. $\beta$-actin was chosen as the internal reference; the upstream primer F2 was 5'-TCGTGCGTGACA TTAAGGAG-3' and the downstream primer R2 was 5'-ATG CCAGGGTACATGGATTG-3'. The target gene and internal reference gene were amplified using an ABI 7500 Real-Time PCR instrument (Applied Biosystems, Foster City, CA, USA). The thermal cycling conditions were set as $50^{\circ} \mathrm{C}$ for $2 \mathrm{~min}$, $95^{\circ} \mathrm{C}$ for $10 \mathrm{~min}$ and then 40 cycles of $95^{\circ} \mathrm{C}$ for $5 \mathrm{~min}, 94^{\circ} \mathrm{C}$ for $15 \mathrm{sec}, 60^{\circ} \mathrm{C}$ for $30 \mathrm{sec}$ and $72^{\circ} \mathrm{C}$ for $30 \mathrm{sec}$. The specimens with the highest content were diluted by 1:1, 1:10, 1:100, 1:1,000, 1:10,000, respectively, in order to perform the amplification by PCR and standard curves were drawn. All experiments for each sample were performed in triplicate.

Statistical analysis. Statistical analysis was conducted using SPSS software, version 17.0 (SPSS Inc., Chicago, IL, USA). The measurement data are represented as mean \pm standard deviation. The means between multiple groups were compared by single factor analysis of variance. The least significant difference method was applied in pairwise comparisons.

\section{Results}

Comparison of IR indices among the groups. The IR index in the rats of the blank control group was $25.85 \pm 3$.66. According to the standards used when establishing the groups, the rats fed with a high fat, high sugar and high protein diet whose IR indices were $>36.83$ were regarded as the experimental group. The remainder of the rats fed with a high fat, high sugar and high protein diet were regarded as the high fat control group. The fasting blood glucose and insulin values of the rats were measured at the end of the experiment in order to calculate the IR index (Table I).

Morris water maze test. In the Morris water maze test, results for the average escape latency period of rats are presented in Table II. The average escape latency to find the platform for the experimental group rats was significantly higher than that 
Table I. Comparison of insulin resistance (IR) index in the three groups of rats at the end of the experiment (mean \pm standard deviation).

\begin{tabular}{lccc}
\hline Group & IR index & F & P-value \\
\hline Blank control & $25.85 \pm 3.66$ & 160.158 & $<0.001$ \\
High fat control & $25.51 \pm 4.10$ & & \\
Experimental & $57.46 \pm 6.75$ & & \\
\hline
\end{tabular}

$\mathrm{P}<0.01$ for the experimental group compared with the blank control and high fat control groups.

Table II. Comparison of the average escape latency period for rats to find the platform in the Morris water maze (mean \pm standard deviation).

\begin{tabular}{lccc}
\hline Group & Latency period (sec) & F & P-value \\
\hline Blank control & $53.63 \pm 14.94$ & 23.416 & $<0.001$ \\
High fat control & $57.19 \pm 11.22$ & & \\
Experimental & $93.56 \pm 20.04$ & & \\
\hline
\end{tabular}

$\mathrm{P}<0.01$ for the experimental group compared with the blank control and high fat control groups.

Table III. Comparison of the results of BACE1 detection by ELISA (mean \pm standard deviation).

\begin{tabular}{llll}
\hline Group & BACE1 $(\mathrm{ng} / \mathrm{ml})$ & F & P-value \\
\hline Blank control & $528.72 \pm 11.72$ & 82.207 & $<0.001$ \\
High fat control & $531.88 \pm 10.23$ & & \\
Experimental & $577.72 \pm 9.34$ & & \\
\hline
\end{tabular}

$\mathrm{P}<0.01$ for the experimental group compared with the blank control and high fat control groups. BACE1, $\beta$-site APP-cleaving enzyme 1; ELISA, enzyme-linked immunosorbent assay.

for the blank control and high fat control groups $(\mathrm{P}<0.01)$. The number of times the rats crossed the platform in the experimental group was significantly decreased compared with that in the high fat control group $(\mathrm{P}<0.01)$. There was no difference in the number of crossings between the rats in the high fat control group and those in the blank control group ( $\mathrm{P}>0.05$; data not shown).

Immunohistochemical results. The immunohistochemical results demonstrated that a slight deposition of $A \beta$ was present in the brains of rats in the high fat control group, while the $A \beta$ deposition was evident in the brains of rats in the experimental group. The deposition could be observed both inside and outside the cells. However, the presence of senile plaques due to the aggregation of $A \beta$ dispersed within the hippocampus was not observed (Fig. 1).
Table IV. Comparison of the detection results of BACE1 by western blot assay (mean \pm standard deviation).

\begin{tabular}{llcl}
\hline Group & BACE1 & F & P-value \\
\hline Blank control & $0.37 \pm 0.07$ & 11.314 & $<0.001$ \\
High fat control & $0.45 \pm 0.09$ & & \\
Experimental & $0.79 \pm 0.14$ & & \\
\hline
\end{tabular}

$\mathrm{P}<0.01$ for the experimental group compared with the blank control and high fat control groups. BACE1, $\beta$-site APP-cleaving enzyme 1; ELISA, enzyme-linked immunosorbent assay.

Table V. Comparison of the results of BACE1 detection by RT-qPCR (mean \pm standard deviation).

\begin{tabular}{lccc}
\hline Group & BACE1 & F & P-value \\
\hline Blank control & $0.368 \pm 0.099$ & 9.833 & $<0.0001$ \\
High fat control & $0.388 \pm 0.100$ & & \\
Experimental & $0.530 \pm 0.093$ & & \\
\hline
\end{tabular}

$\mathrm{P}<0.01$ for the experimental group compared with the blank control group and the experimental group compared with the high fat control group. BACE1, $\beta$-site APP-cleaving enzyme 1; RT-PCR, reverse transcription-quantitative polymerase chain reaction.

Results of BACE1 detection by ELISA. ELISA results evaluating the levels of BACE1 are shown in Table III. The amount of BACE1 in the hippocampus of rats in the experimental group was significantly higher than that in the blank control and high fat control groups $(\mathrm{P}<0.01)$.

Results of BACE1 detection by western blotting. The expression of BACE1 in the cerebral tissue of the rats in each group was detected by western blotting. It was observed that BACE1 was expressed in the cerebral tissue of the rats in each group. The expression of BACE1 in the experimental group was significantly higher than that in the other two groups $(\mathrm{P}<0.01$; Table IV).

Results of BACE1 detection by RT-qPCR. The mRNA expression level of BACE1 was calculated by RT-qPCR analysis. The expression level of BACE1 mRNA in the hippocampus of rats in the experimental group was significantly higher than that in the blank control and high fat control groups $(\mathrm{P}<0.01$; Table V).

\section{Discussion}

$\mathrm{AD}$ is a degenerative disease of the nervous system associated with multiple factors. The pathogenesis of AD is not very clear; however, the amyloid protein cascade hypothesis is an acceptable hypothesis to a large number of scholars at present (9). It considers that the deposition of $A \beta$ is the originating factor of AD. Certain studies have shown that the cerebral glucose metabolic rate and energy production are significantly decreased in 

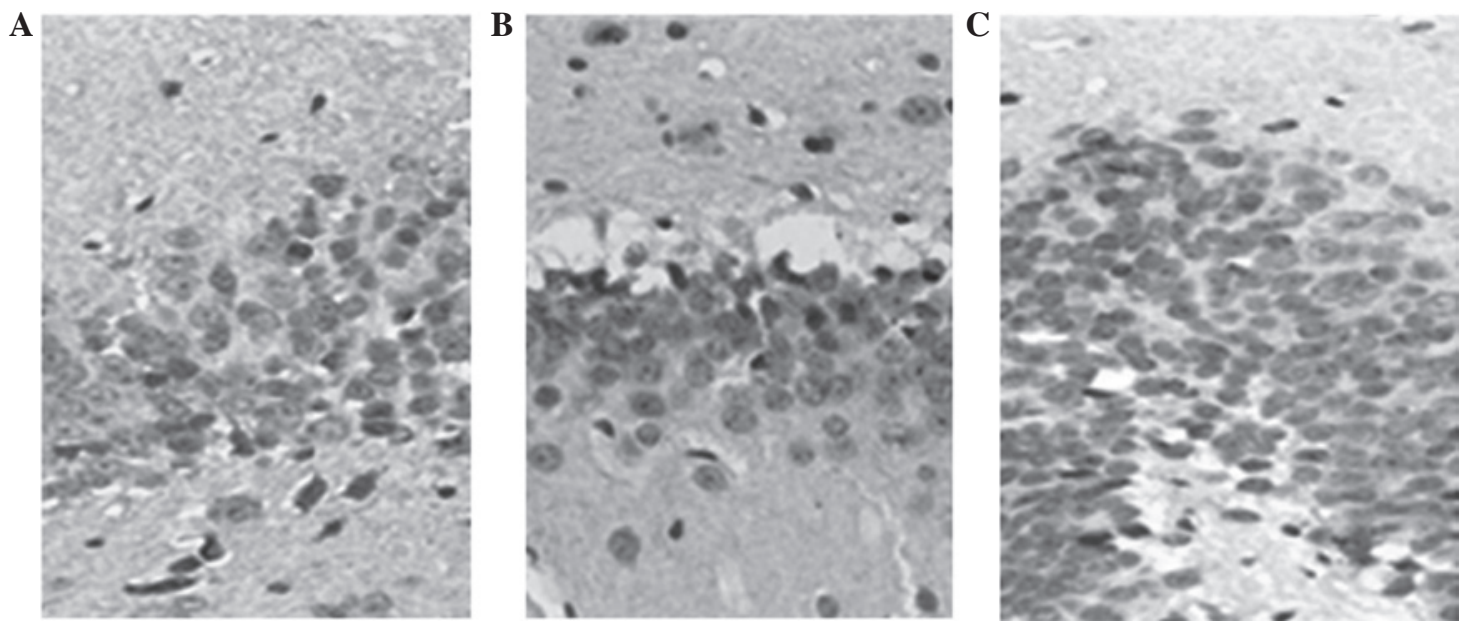

Figure 1. Immunohistochemical results for amyloid $\beta$ in the three groups. No senile plaques were observed in the three groups. (A) Blank control, (B) high-fat control and (C) experimental groups.

patients with $\mathrm{AD}(10,11)$. Dysfunction of insulin and the insulin receptor signal transduction system plays an important role in the pathological process of AD (12). This is associated with the cerebral glucose oxidative metabolism and energy production (13), the over-phosphorylation of $\tau$ protein (14), cell apoptosis in easily affected brain regions (15) and the aggregation of $A \beta$ in cells (16), followed by a deficiency of energy metabolism, acetylcholine imbalance and neuronal apoptosis that eventually lead to dementia.

BACE1 is a $\beta$ secretory enzyme, and a rate-limiting enzyme in the production of $A \beta$. It degrades $\beta$ amyloid precursor protein (APP) to form A $\beta$ (17). A large number of studies have demonstrated that inhibition of the expression of BACE1 may control AD $(18,19)$. Therefore, there appears to be a close association between BACE1 and AD.

STZ is a nitrosourea derivative. It blocks the autophosphorylation of the insulin receptor and intrinsic tyrosine kinase activity in the main center of the brain and influences the function of the insulin receptor. Once the insulin/insulin receptor system is destroyed, the metabolism of nerve cells in the cerebral cortex and hippocampus is likely to be affected (20). The disturbance of glucose metabolism leads to a reduction of ATP production in affected cells. The intracerebroventricular injection of small dose of STZ can lead to the reduction of cerebral insulin levels while not affecting insulin in the peripheral circulation. This can induce IR in the central nervous system and the development of progressive neurodegenerative disease, leading to the development of dementia (21). The STZ-induced animal model has many similarities with the clinical manifestations and pathological changes of $\mathrm{AD}$. The animal model has become an important model for performing 'insulin-AD' relevant research $(21,22)$. In the present study, the rats were fed with a high fat, high sugar and high protein diet for 12 weeks to induce IR in the rats of the experimental group. An intracerebroventricular injection of a small dose of streptozotocin was then administered. The model is consistent with the clinical situation. Following the establishment of the model, the learning changes of the rats were detected by Morris water maze test in each group. The latency period in searching for the platform in the rats in the experimental group was significantly longer than that in the high fat control group $(\mathrm{P}<0.01)$. There was no significant difference in latency period between the high fat control and control groups $(\mathrm{P}>0.05)$. The number of times the rats in the experimental group crossed the platform was significantly decreased compared with that in the high fat control group $(\mathrm{P}<0.01)$; however, there was no difference between the rats in the high fat control and blank control groups $(\mathrm{P}>0.05)$. This suggests that the model of dementia induced by IR was successful.

Histopathological examination of the brains of rats in the experimental group indicated that there were no pathological changes characteristic of AD. The results of ELISA, western blotting and RT-qPCR revealed that the hippocampal content of the BACE1 enzyme and the expression of BACE1 mRNA in the rats of the experimental group were higher compared with those in the high fat control and blank control groups, and the difference was statistically significant $(\mathrm{P}<0.01)$. However, there was no significant difference in BACE1 level between the high fat control and blank control groups $(\mathrm{P}>0.05)$. Thus it may be assumed that IR led to the increase of BACE1 expression in insulin-resistant rats, and was involved in the pathogenesis of $\mathrm{AD}$. However, for the rats in the experimental group, no characteristic pathological changes of $\mathrm{AD}$ were detected in the brain by histopathological analysis. It is speculated that the possible reason was that it is a comparatively long process from the occurrence of IR to the appearance of the characteristic pathology of AD. Considering the body as a whole, in addition to the BACE1 enzyme which increases $A \beta$ levels, there are also enzymes that can clear A $\beta$. Both are able to maintain a dynamic balance to a certain extent. Therefore, BACE1 is just one of the enzymes influencing the metabolism of $A \beta$, and may not necessarily lead to a pathological change of a certain extent at a specific time.

In addition, studies have shown that the occurrence of SAD is caused by multiple factors, such as redundant $A \beta$ production, hyperphosphorylation of $\tau$ protein, generation of free radicals and oxidative stress damage (23-25), inflammatory factors, individual biological characteristics, past disease history/complications, habits, dietary habits, contact with certain substances or environments and social/psychological factors. 
In summary, the present study found increased expression levels of BACE1 in the brain tissue of rats with IR. It also indicated that IR is involved in the pathogenesis of AD from a single aspect. The specific mechanism of IR in the pathogenesis of AD requires further study.

\section{References}

1. Chai GS, Duan DX, Ma RH, et al: Humanin attenuates Alzheimer-like cognitive deficits and pathological changes induced by amyloid $\beta$-peptide in rats. Neurosci Bull 30: 923-935, 2014.

2. Wang S and Jia J: Promoter polymorphisms which modulate BACE1 expression are associated with sporadic Alzheimer's disease. Am J Med Genet B Neuropsychiatr Genet 153B 159-166, 2010.

3. Pera M, Alcolea D, Sánchez-Valle R, et al: Distinct patterns of APP processing in the CNS in autosomal-dominant and sporadic Alzheimer disease. Acta Neuropathol 125: 201-213, 2013.

4. Tang J, Pei Y and Zhou G: When aging-onset diabetes is coming across with Alzheimer disease: Comparable pathogenesis and therapy. Exp Gerontol 48: 744-750, 2013.

5. Yarchoan M and Arnold SE: Repurposing diabetes drugs for brain insulin resistance in Alzheimer disease. Diabetes 63: 2253-2261, 2014

6. Ghasemi R, Dargahi L, Haeri A, Moosavi M, Mohamed Z and Ahmadiani A: Brain insulin dysregulation: Implication for neurological and neuropsychiatric disorders. Mol Neurobiol 47: 1045-1065, 2013

7. Zhang T and Liu Y: Insulin resistance animal model induced by high-energy dietary. Shi Yan Dong Wu Ke Xue 25: 48-51, 2008 (In Chinese)

8. Bromley-Brits K, Deng Y and Song W: Morris water maze test for learning and memory deficits in Alzheimer's disease model mice. J Vis Exp 53: 2920, 2011.

9. Matsuzaki K: How do membranes initiate Alzheimer's Disease? Formation of toxic amyloid fibrils by the amyloid $\beta$-protein on ganglioside clusters. Acc Chem Res 47: 2397-2404, 2014.

10. Amaral AI, Teixeira AP, Håkonsen BI, Sonnewald U and Alves PM: A comprehensive metabolic profile of cultured astrocytes using isotopic transient metabolic flux analysis and C-labeled glucose. Front Neuroenergetics 3: 5, 2011.

11. Charron MJ and Vuguin PM: Lack of glucagon receptor signaling and its implications beyond glucose homeostasis. J Endocrinol 224: R123-R130, 2015.
12. Correia SC, Santos RX, Perry G, Zhu X, Moreira PI and Smith MA: Insulin-resistant brain state: The culprit in sporadic Alzheimer's disease? Ageing Res Rev 10: 264-273, 2011.

13. Lannert $\mathrm{H}$ and Hoyer S: Intracerebroventricular administration of streptozotocin causes long-term diminutions in learning and memory abilities and in cerebral energy metabolism in adult rats. Behav Neurosci 112: 1199-1208, 1998.

14. Lesort M, Jope RS and Johnson GV: Insulin transiently increases tau phosphorylation: Involvement of glycogen synthase kinase- $3 \beta$ and Fyn tyrosine kinase. J Neurochem 72 : 576-584, 1999.

15. Jin Y, Fan Y, Yan EZ, Liu Z, Zong ZH and Qi ZM: Effects of sodium ferulate on amyloid- $\beta$-induced MKK3/MKK6-p38 MAPK-Hsp27 signal pathway and apoptosis in rat hippocampus. Acta Pharmacol Sin 27: 1309-1316, 2006.

16. Kondo M, Tokuda T, Itsukage M, et al: distribution of amyloid burden differs between idiopathic normal pressure hydrocephalus and Alzheimer's disease. Neuroradiol J 26: 41-46, 2013.

17. Chu J, Zhuo JM and Praticò D: Transcriptional regulation of $\beta$-secretase- 1 by $12 / 15$-lipoxygenase results in enhanced amyloidogenesis and cognitive impairments. Ann Neurol 71: 57-67, 2012.

18. Chiocco MJ and Lamb BT: Spatial and temporal control of age-related APP processing in genomic-based $\beta$-secretase transgenic mice. Neurobiol Aging 28: 75-84, 2007.

19. Vassar R: BACE1 inhibitor drugs in clinical trials for Alzheimer's disease. Alzheimers Res Ther 6: 89, 2014.

20. Sonkusare S, Srinivasan K, Kaul C and Ramarao P: Effect of donepezil and lercanidipine on memory impairment induced by intracerebroventricular streptozotocin in rats. Life Sci 77: 1-14, 2005.

21. de la Monte SM, Tong M,Lester-Coll N, Plater M Jr and Wands JR: Therapeutic rescue of neurodegeneration in experimental type 3 diabetes: Relevance to Alzheimer's disease. J Alzheimers Dis 10: 89-109, 2006.

22. Grünblatt E, Salkovic-Petrisic M, Osmanovic J, Riederer P and Hoyer S: Brain insulin system dysfunction in streptozotocin intracerebroventricularly treated rats generates hyperphosphorylated tau protein. J Neurochem 101: 757-770, 2007.

23. Chabrier MA, Blurton-Jones M, Agazaryan AA, Nerhus JL, Martinez-Coria $\mathrm{H}$ and LaFerla FM: Soluble A $\beta$ promotes wild-type tau pathology in vivo. J Neurosci 32: 17345-17350, 2012.

24. Vassar R: $\beta$-secretase, APP and A $\beta$ in Alzheimer's disease. Subcell Biochem 38: 79-103, 2005.

25. Yan R and Vassar R: Targeting the $\beta$ secretase BACE1 for Alzheimer's disease therapy. Lancet Neurol 13: 319-329, 2014. 